$\begin{array}{ll}\text { Italique } & \text { Italique } \\ \text { Poésie italienne de la Renaissance }\end{array}$

XVIII | 2015

Varia

\title{
Sofonisbe e Susanne: ragioni metriche nella genesi della tragedia rinascimentale
}

\section{Renzo Cremante}

\section{(2) OpenEdition}

\section{Journals}

\section{Edizione digitale}

URL: http://journals.openedition.org/italique/406

DOI: $10.4000 /$ italique.406

ISSN: 1663-4438

\section{Editore}

Librairie Droz

\section{Edizione cartacea}

Data di pubblicazione: 1 ottobre 2015

Paginazione: 63-88

ISBN: 978-2-600-01900-2

ISSN: 1423-3983

\section{Notizia bibliografica digitale}

Renzo Cremante, "Sofonisbe e Susanne: ragioni metriche nella genesi della tragedia rinascimentale», Italique [Online], XVIII | 2015, online dal 01 octobre 2017, consultato il 07 mai 2019. URL : http://

journals.openedition.org/italique/406 ; DOI : 10.4000/italique.406 
Renzo CREM A T T

SOFONISBE E S S A N NE:

R A G I O N M E T R I C H E

NELLA GENES I

D E L L A TRAGED I R INASCIM E N T A L E 



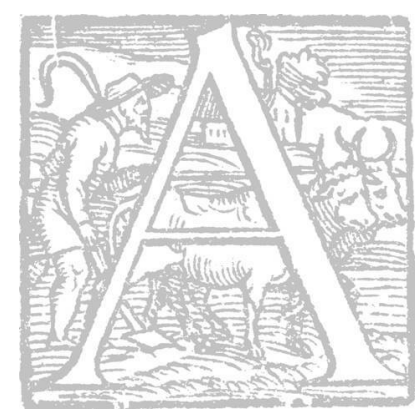

lle ragioni metriche spetta, com'è noto, un ruolo di preminente rilievo nella poetica tragica e nella genesi ed elaborazione della Sophonisba di Giovan Giorgio Trissino, «il primo tentativo post-classico attuato in una letteratura europea di drammatizzare una storia antica in una forma che imita la tragedia classica, ma che è resa in lingua volgare». ' Le rivoluzionarie proposte strutturali, formali, linguistiche, ortografiche, nella fattispecie metriche, di quel memorabile archetipo, segnano una dirompente novità e assoluta, programmatica incompatibilità rispetto a qualsivoglia prodotto del consumo letterario o teatrale comecchessia divulgato, a cavallo tra Quattro e Cinquecento, sia in latino sia in volgare, sotto l'etichetta equivoca, generica e approssimativa di tragedia. Basti un primo, pur sommario confronto, quanto scontato tanto inevitabile, con un altro testo ispirato alla medesima fabula e ugualmente dedotto, con non minore fedeltà, dalla fonte liviana: ${ }^{2}$ la Sophonisba di Galeotto del Carretto, composta una dozzina d'anni prima dell'omonima tragedia trissiniana ma pubblicata postuma soltanto nel I546, ${ }^{3}$ sull'onda del crescente favore editoriale, ma oramai anche scenico, che il nuovo genere andava riscuotendo in quel giro d'anni. ${ }^{4}$ Si noti, per altro, che la denominazione di 'tragedia' compare soltanto nel frontespizio della stampa e nella lettera di dedica ad Alberto del Carretto, datata «Di Casale in Monferrato del MDXLV» e sottoscritta dal curatore, Niccolò Franco; il quale, accomunando l'opera del poeta monferrino, morto nel I530, alla tragedia di Trissino, pubblicata per la prima volta nel Is 24 e già più volte ristampata, ${ }^{5}$ mal dissimula l'insolente proposito di metterne in tal modo in discussione il primato, nonché di sfruttarne il successo. ${ }^{6}$ Nella lettera di dedica indirizzata a Isabella d'Este Gonzaga, marchesana di Mantova, datata 22 marzo I502 e allegata anch'essa alla stampa, l'autore non parla propriamente di tragedia, ma di «opera mia continuata, la qual per una volta sarà in satisfatione de le mie rime, che le soleva mandare, e del tempo interrotto in scriverle al solito costume» (c. 5v). ${ }^{7}$ Ma è il Prologo, giusta la connaturata intonazione classicheggiante, che s'innalza a dichiarare: «Melpomene mi sprona a sonar versi / Con mesti accenti, e tragico boato, / E dir di Sophonisba $i$ casi aversi / Et il suo acerbo, e miserabil fato»; mentre 
prende alla fine congedo dal pubblico con l'invito ad ascoltare "Quel che ba da dirgli la dogliosa rima» (c. 6r).

Trasferendo alla materia classica, storica e profana, e all'ambito cortigiano il paradigma consolidato della sacra rappresentazione e arricchendolo di un brevissimo Prologo e di un Argomento, l'«opera [...] continuata» si compone, se non bo contato male, di 44 sequenze fra dialogiche, se di dialogo si può propriamente parlare, e monologiche (se ne contano, di queste, ben I7), ${ }^{8}$ per un totale di 2373 versi: 281 in più della tragedia di Trissino; con una varietà e pluralità di azioni, di tempi ( $i$ piu lustri che abbracciano l'intero arco cronologico della seconda guerra punica), ${ }^{9}$ e di luoghi (dalla Spagna all'Africa, dall'Abruzzo alla Sicilia, da Cadice a Cartagine, a Cirta). Curiosa 'tragedia'! Sia pure l'intera architettura dell'opera costruita in funzione della catastrofe e dell'atto supremo della protagonista, vero è che nell'economia della narrazione il racconto del «miserevole caso» di Sofonisba occupa tanto meno spazio di quello riservato, poniamo, ai «notabili gesti di Scipione in Affrica dopo la superata Spagna», o a «l'infortunato successo» di Siface, per citare ancora la lettera dedicatoria (c. sr). All'eponima eroina cartaginese sono riservate non piu di quattro battute, I7 sole ottave, 136 versi in tutto, mentre il $65 \%$ dell'intero tessuto dialogico è occupato dalle monocrome tiritere di Scipione, di Lelio e di Asdrubale figlio di Gisgone, il padre di Sofonisba (escluso, quest'ultimo, dalla tavola della «persone che parlano ne la favola» trissiniana).

Dovendo la narrazione farsi carico anche dei raccordi scenici, accompagnare i personaggi nei loro incessanti andirivieni, navali e terrestri, da un capo all'altro del teatro mediterraneo della guerra, le sequenze, sul modello delle sacre rappresentazioni, sono introdotte da circostanziate didascalie, del tipo: «Asdruballe, figlinolo di Gisgone, essendo a Gade dice alli suoi» (c. Iov); «Scipione andando verso il real palazzo dice per camino a' suoi compagni» (c. I6r); «Lelio venendo dal porto con un suo compagno, dice» (c. 24r); "Scipione giunto in Sicilia per passare in Affrica da sé dice così (c. 27v); "Sophonisba ingenocchiata avanti a Massinissa, pigliandolo per mano gli dice» (c. 39r), ecc.; ${ }^{\text {10 }}$ e sono inframmezzate da 28 interventi di un Coro, la cui qualità non è altrimenti specificata. Il Coro, non interloquendo mai direttamente con $i$ personaggi, riveste la funzione drammaturgica, ma anche, al contempo, didascalica, di delucidare, riepilogare e commentare lo svolgimento dell'azione: con più commossa partecipazione, in una prospettiva 
sempre più scopertamente lirica, nella parte conclusiva, a partire dall'annuncio dell'imminente morte di Sofonisba, quando anche, in un punto, si divide in due semicori (cc. 49v-sor). I $D i$ contenuto moraleggiante, quale poi si ritroverà in tanti cori di tragedie 'regolari' cinquecentesche, sono due cori dedicati, rispettivamente, all'Invidia $(\text { c. } 27 r)^{\mathrm{I} 2}$ e alla Fede (c. 33rv); e il pensiero corre subito ai cori delle tragedie giraldiane. ${ }^{\mathrm{I}}$

Con due sole eccezioni, compresa l'ottava finale del Coro che suggella la tragedia (c. 52v), e come avviene, di norma, nei cosiddetti drammi "mescidati" del teatro cortigiano e rinascimentale, la distinzione fra parti dialogiche e parti corali, e dunque, almeno nelle intenzioni, fra recitazione e canto, è anche di ordine metrico. Sull'esempio, infatti, della sacra rappresentazione, al discorso continuato dei dialoghi e dei monologhi (che insieme costituiscono quasi il $77 \%$ del testo), è riservato l'impiego esclusivo dell'ottava rima. Le battute sono in genere brevi, perlopiù comprese in una misura che va da una a quattro ottave, ma può eventualmente estendersi fino a sette (come nella citata risposta di Sofonisba al Servo mandato da Massinissa con la coppa del veleno, cc. $47 v-48 v)$, una volta a otto (cc. $2 I v-22 v)$, eccezionalmente fino a quindici ottave, per un totale di I20 versi (cc.37r-39r), là dove «Massinissa, con alcuni suoi, giunto alla porta di Cirtha, narra il progresso de la guerra». Nei dialoghi l'ottava non è, di norma, spezzata; soltanto in numerate eccezioni si riscontra una divisione fra il sestetto a rime alternate e il distico baciato finale (cc. $8 v$, I $5 v, I 8 v$-Igr, 24v), mentre in un'unica occorrenza la divisione cade giusto a mezzo dell'ottava, quattro versi per parte (c. 27r). Una volta soltanto infine, dialogando Scipione e Siface, a essere spezzata, fuor di cesura, è la misura dell'endecasillabo (c. 24v). Vale forse anche la pena di sottolineare come il periodo sintattico, schematico e lineare, soltanto qua e là intralciato da rari enjambement, tenda più spesso ad abbracciare l'intera misura della stanza, oppure a procedere senza sussulti, con cadenza regolare, di quattro in quattro versi, con prevalente effetto di staticità e monotonia, confermata anche dalla partitura rimica, dove prevalgono le rime facili, desinenziali, suffissali (quante rime in -are, -ore, -ato ecc..), non senza sparse occorrenze di rime equivoche, derivative, inclusive, ricche, di norma piane, solo eccezionalmente sdrucciole o tronche. Può anche capitare, con una certa frequenza, che le medesime rime, o perfino l'intero sistema delle medesime parole-rima, siano ripercosse a distanza 
di pochissime ottave, ${ }^{\mathrm{I}}$ o anche in ottave contigue (cfr. per esempio le cc. I5r, I9v, 22r, 24v, 27v, 37v-38r, 39rv, 43v, 5IV-52r), con effetti talora, non so quanto consapevoli, di coblas capcaudadas. ${ }^{\mathrm{Is}}$

Di contro all'uniformità metrica e ritmica delle ottave dialogiche o narrative, $i$ cori registrano una considerevole varietà morfologica di metri lirici, in genere brevi, da 8 a 16 versi, omometrici o eterometrici, endecasillabi e settenari, variamente rimati, sotto forma di madrigali, canzoncine, pseudosaffiche con rimalmezzo, di norma, in quinta sede, secondo lo schema (a) $\mathrm{B}(\mathrm{a}) \mathrm{B}(\mathrm{b}) \mathrm{Cc}$, essendo c quinario (cc. I $4 \mathrm{v}$, $24 \mathrm{r}$ e 28v) $;^{16}$ ma non mancano, come si è accennato, strambotti in veste di ottava toscana, anche composti di soli versi settenari (cc. IsvI $6 r$ ), o con sestetto di settenari ed endecasillabi alternati e coppia finale di endecasillabi, secondo lo schema $\mathrm{aBaBaBCC}$ (c. IIv); un capitoletto moraleggiante in terza rima di $37 \mathrm{vv}$., con verso di chiusura, tutto incentrato, come abbiamo già ricordato, sulla celebrazione della Fede, parola-chiave replicata otto volte per anafora (c. 33rv); una serie di II endecasillabi 'frottolati' con rimalmezzo, al modo del gliuommero (c. 3 r r), metro collaudato nella poesia rappresentativa cortigiana; ${ }^{17} e$ persino un sonetto, con schema ABBA. ABBA. CDE. CDE (c. 27r). Quando il Coro si divide in due semi-cori, anche l'ode, se vogliamo servirci di questa terminologia nella sua accezione cortigiana e primocinquecentesca, si divide, replicandoli 4 volte, in due gruppi, metricamente collegati, di s, rispettivamente e 4 versi, con schema di rime $\mathrm{ABCcB} e \mathrm{ADdA}$.

Per la storia del verso drammatico più importa, naturalmente, la presenza - concentrata nella parte finale della tragedia, quando l'azione si sposta a Cirta, fino al sacrificio della protagonista - di tre cori, rispettivamente, di 38, 3 I e 48 endecasillabi non rimati, in netta prevalenza a maiori (cc. 40rv, 48v-49r e 5Irv), usati forse per la prima volta in un testo drammatico, sia pure in funzione lirica, non già dialogica. Ė ben probabile, a mio giudizio, che Galeotto avesse presenti gli endecasillabi saffici dei cori delle tragedie senecane, e non soltanto per quanto concerne la versificazione. Ma conviene sottolineare, soprattutto, l'intonazione spiccatamente patetica di queste primissime attestazioni di endecasillabi liberati dall' accordo obbligato delle desinenze. Mi limito a segnalare quest'unico riscontro, estratto dal penultimo coro (c. SIV): 
Ahi miser Massinissa, hor bene il sai, Bene il mostri nel duol, ch'ogni riposo Ti circonscrive e l'allegrezza insieme, Mentre sparir da gli occhi tuoi si vede Quella ch'apena fu dal tuo desio Con nodo marital teco congiunta, Che morte sì crudel indi l'ha sciolta $[\ldots]$

Piagni misero dunque, e gli occhi in fiumi Cangia se puoi, perché a l'ingiusto male Porgano pianto con perpetua vena.

L'istituzione trissiniana del verso sciolto, traslocato dai pausati margini lirici del coro al centro vitale dell'azione e al dialogo continuato dei personaggi, non potrà non tener conto di un precedente siffatto e delle sue potenziali applicazioni al registro, in particolare, affettuoso della dialogicità tragica, ${ }^{\mathrm{I}}$ a una tragedia "passionale», quale lo stesso Trissino, sulla scorta della classificazione aristotelica, rubricherà la sua Sophonisba nella quinta divisione dell'Arte Poetica: ${ }^{19}$ se è vero che l"inventore" di un genere metrico procede, di norma, da fattori già sperimentati e sparsamente presenti in altri contesti della tradizione prossima o remota, ma per trasformarli radicalmente, per "grammaticalizzarli" e ricomporli in un'inedita struttura unitaria, rendendoli in tal modo necessari e funzionali a nuovi contesti, a nuove strutture, a nuove esigenze espressive. Soccorrono ancora, nel caso di Galeotto, di là da moduli convenzionali ed echi danteschi, petrarcheschi o petrarchistici di immediata riconoscibilità, minimi esempi di anticipato, occasionale collaudo, per esempio, di commiserazioni o manifestazioni di dolore, più o meno topiche, che ritroveremo nella tragedia trissiniana, del tipo: "Abi misero Siphace, e tu che fai? / Come non piagni se sei giunto a tale, / Che con l'bonor la libertate hai spenta?» (c. 40v, cfr. Trissino, Sof., 294-95: "Ahi misero Syphace, / Dove, dove n'andrai, dove mi lasci》; e I203: "Ecco, siete ridotto a caso tale / Ch'io non vi posso dare alcuno aiuto»: sono parole di Sofonisba, rispettivamente, e di Scipione); «Ben fora ogni pietà da gli occhi vostri / Sbandita al tutto» (c. 48v, cfr. Trissino, Sof., 296-97: "Qual spirto al mondo è di pietà sì nudo / Che mirando hor costei tenesse il pianto»); "E se potesse anchor morirne insieme, / Il morir le saria più grata vita» (c. sir, cfr. Trissino, Sof., I 590-9I: "Ver è che più le aggradiria il morire / Se ne la morte 
non prendea marito»); "Che tanto non potrai piagner tra noi / Quanto n'hai dal dolor giusta cagione» (c. 5Iv, cfr. Trissino, Sof., 30I: «Giusta cagione a lacrimar vi move»).

Entro un pur ampio ventaglio di applicazioni, compreso l'impiego nei dialoghi della terza al posto dell'ottava rima, la Sophonisba di Galeotto del Carretto può bene illustrare la varia fisionomia polimetrica di molti testi drammatici, sia tragici, sia comici, sia allegorici, composti negli ultimi decenni del Quattrocento e nei primi del Cinquecento, eventualmente messi in scena, e affidati a una diffusione manoscritta o a stampa che si prolunga ben oltre $i$ termini cronologici fissati dall'apparizione e dalla circolazione delle prime commedie e tragedie 'regolari' d'imitazione classica. ${ }^{20}$ La polimetria, del resto, non sempre è circoscritta alle sole parti liriche, là dove sia prevista la presenza del coro. Non è privo d'interesse, a questo riguardo, un testo di tutt'altra natura e che anzi sembra deliberatamente contrapporsi ad azioni teatrali di argomento profano e storico come quelle che abbiamo appena esaminato: la Sosanna di Tiburzio Sacco. ${ }^{2 \mathrm{I}}$

Scarsissime le notizie sull'autore, un frate domenicano di Busseto vissuto a cavallo fra XV e XVI secolo; non si conoscono altre sue opere. Alla genesi e alla destinazione di questa «tragedia nova», come la designa il frontespizio, accenna esplicitamente la lettera di dedica della prima edizione, sottoscritta dall'autore, datata Venezia, 7 marzo I524, e indirizzata a un correligionario, il padre Felice Taverna, priore del convento milanese delle Grazie, con l'auspicio che egli sappia ergersi come «scudo sodissimo» contro «le velenose saette de' pallidi detrattori» e come «provido et indefesso defensore» contro gli «abbagliatori» della Sosanna:

Non saranno dunque e Religiosi con diritta conscientia colpati, se alle volte, et specialmente nel tempo carnevalesco, interporranno qualche honesto solazzo alle insoportabili fatiche claustrali; anzi pur lodati, quando il loro remisso studio in ocio spirituale ispenderassi. Ma qual più lodevole ricreamento fia a' cappucciati, ch'il rappresentare qualche historia dil vecchio, over nuovo testamento? ${ }^{22}$

In apertura il Prologo, in ottava rima, si preoccupa di informare $i$ «cappucciati» spettatori che essi non saranno chiamati ad assistere alla rappresentazione di una commedia, a uno spettacolo di «giocosa e fenta poesia): 
Mio costume non è di tal bugia.

Vera storia vedret' antica e degna,

Ch'a nui frati mi par che più convegna. ${ }^{23}$

Quella che si propone loro è una storia tragica, prelevata tuttavia, prosegue il Prologo, non già dal serbatoio tradizionale della storia antica, greca o romana (sono elencati una decina di autori, a cominciare da Livio, la fonte appunto delle due Sophonisbe: «Non già storia di Livio Padoano»), ma dalla storia sacra, dall'autorità biblica del Libro del profeta Daniele (I3, I-64):

Né Herodoto, Laertio, né Appiano,

Né altr'historico greco, né latino

Giamai discusse questo, né Poeta,

Ma sol si legg' in Danïel Profeta. ${ }^{24}$

Anzi, proprio come accade nel racconto della Bibbia seguito passo passo e tradotto in più punti alla lettera, a «sciorr' il nodo ${ }^{25}$ / Ch'occupa l'innocentia di Sosannas (vv. Iog-Io), nella scena IV del IV atto interviene la persona stessa del profeta, nella funzione teatrale, si direbbe, di deus ex machina: un altro curioso esempio di interferenza fra materia sacra e modelli classici. L'argomento, del resto, era già entrato nel repertorio delle sacre rappresentazioni. ${ }^{26}$

L'episodio biblico, circoscritto entro ben ristretti confini spaziali e temporali e contenuto nella misura di 64 versetti, privo di per sé di potenziali dilatazioni fabulistiche, mal poteva prestarsi all'elaborazione di un intreccio complesso e articolato. La Sosanna si distingue anche per la notevole brevità, come il Prologo, consapevole che «ciascun che la misura eccede / Suole il più delle fiate esser biasmato», sente il bisogno di giustificare con argomenti di carattere più generale:

Sarrà questo spettacol assai breve,

Ch'ogni prolissità sempre rincresce.

Un bel canto si fa noioso e greve

Quando ch'oltre il dover lungo riesce.

D'una bella pittura se riceve,

Per mirar troppo, ch'el piacer decresce.

Spiace un longo, quantunque dolce, suono,

$\mathrm{E}$ sol diletta quel ch'è puoco e buono. ${ }^{27}$ 
Divisa in cinque atti $e$ in scene progressivamente numerate, la tragedia si sviluppa per un totale di soli ${ }_{12} 38$ versi, ${ }^{28}$ uno in meno della Rosmunda di Giovanni Rucellai, di gran lunga la più breve e scorciata fra tutte quante le tragedie 'regolari' cinquecentesche, uscita a stampa, postuma, con la data del 27 aprile is 25 e con la cospicua novità, rispetto alla tragedia di Trissino con la quale pure era nata a un parto, ${ }^{29}$ della divisione in atti. ${ }^{30}$ L'alto numero delle scene, $27,{ }^{3 \mathrm{I}}$ comporta, di conseguenza, che esse siano brevi o brevissime: la più lunga, di gs versi (a. II, s. II), la più breve, pur in dialogo, di una sola ottava (a. IV, s. I). Poco meno di un terzo delle scene ammette un solo personaggio, quattro volte a parlare da solo è il servo Siro. Né deve stupire in una tragedia di argomento biblico, in una ierotragedia, come la si sarebbe chiamata alla fine del secolo, la presenza nella tavola dei personaggi, e in ruoli ben caratterizzati, di servi, di famulanti, come qui sono chiamati, ${ }^{32}$ addirittura di un «mal nato» servo archetipico della commedia latina, sia plautina sia terenziana, e dal nome già utilizzato, fra gli altri, da Boiardo nel Timone e da Machiavelli nella Mandragola (come poi nella Clizia). Nell'azione scenica della Sosanna Siro occupa un ruolo niente affatto secondario, ed è proprio lui a suggellarne la conclusione, prendendo congedo dagli spettatori con la rituale formula latina: "Ė questo l'ultim'atto. / Altro non più vedrete! / Vos interim valete / Et plaudite» (a. V, s. VII, 259-62).

Un interessante elemento di novità nella polimetria dei dialoghi è rappresentato dal tentativo di sfruttare la veste metrica, non si dirà per aiutare a delineare meno superficialmente la psicologia dei singoli personaggi, ma almeno per identificarli, per distinguerne la varia tipologia funzionale, pur all'interno di un dettato, con poche eccezioni, complessivamente uniforme, privo di irregolarità o di scarti. Così, Sosanna si esprime per frottole di endecasillabi con la ripercussione ripetuta e ravvicinata della rimalmezzo (di norma in coincidenza con la cesura di settima, ma sporadicamente anche in quinta ed eccezionalmente in quarta sede): il metro del gliuommero, già impiegato da Galeotto del Carretto, come si è accennato, in una sezione però corale della Sophonisba, ${ }^{33}$ ma con più alta frequenza per esempio, in molte parti dialogiche delle Noze de Psiche et Cupidine e del Tempio de amore. ${ }^{34}$ Nacchor e Chaynam, $i$ due Vecchi «giudicanti» dalla stravagante onomastica biblica e che prima insidiano e poi accusano e condannano per adulterio Susanna, si servono dell'ottava rima per 
ordire la loro macchinazione ai danni della protagonista. Nella stringatezza del dialogo le loro battute solo raramente sfiorano e non superano mai la misura di quattro stanze, mentre nella scena II dell'atto II manifestano a Sosanna i loro turpi ardori scambiandosi vicendevolmente le stanze. Dai due Vecchi, poi, l'impiego dell'ottava si estende agli eventuali interlocutori, prima allo stesso profeta Daniele (a. IV, s. IV), poi al Cavaliero e agli Sbirri incaricati di eseguire la sentenza di morte per lapidazione nei loro confronti (a. $V$, s. I e s. III). Ma l'ottava è anche il metro usato di norma da Helchia ed Ethuria, gli sconsolati genitori di Sosanna. E si tratta, in tutti i casi, di ottave piatte e uniformi, che procedono quasi sempre, con cadenza regolare, di distico in distico, divise qua e là dalle necessità del dialogo, che soltanto in rarissime occasioni spezza l'unità del verso, in un solo caso in tre membri (a. I, s. II, v. 92).

Ancora, la figurina arguta e scanzonata del servo Siro - «Io mai conobbi un si bizzar cervello», dice di lui il compagno Cleante (a. V, s. V, v. I77)è contrassegnata da un metro scorrevole e veloce: strofette di tre settenari chiuse da un quinario iniziante pervocale e unito per sinalefe alverso precedente (più raramente, quando il versicolo inizia con consonante, da un quadrisillabo), concatenate per la rima alla strofetta seguente, secondo lo schema $\mathrm{a}^{7} \mathrm{~b}^{7} \mathrm{~b}^{7} \mathrm{c}^{5}, \mathrm{c}^{7} \mathrm{~d}^{7} \mathrm{~d}^{7} \mathrm{e}^{5}$ ecc. ${ }^{35}$ Ed èforse questo il casopiù felice, nella Sosanna, di corrispondenza fra metro epersonaggio, con effetti di notevole immediatezza e vivacità espressiva, in un registro, anche linguistico, dichiaratamente comico (a. I, s. III, vv. I80-99):

L'altr'hieri, perché diedi

Una rispost' altiera,

E certo più che vera,

Al mio patrone,

Oimé, ch'a strangoione

Fécimela mangiare;

Et cominciò cridare

Ad alta voce:

Fuor di la boccha bava

Gettava d'ogni canto;

Non tigre né elefanto

È tanto fiero. 
Valga quest'altro esempio, dove Siro esprime la propria incredulità, incrinata da convenzionali spunti antifemministi, alla notizia che $i$ Vecchi scellerati hanno mosso a Sosanna la falsa, infamante accusa di adulterio (a. III, s. I, vv. I7-32):

Non so se vivo sono,

Son fuori di me stesso.

O gravissim'eccesso

Di Sosanna!

Che fatta sia putanna

Nol posso sopportare,

Et ciò non so pensare

Dove vegna.

Adesso più non regna

Fede tra queste donne;

Per una over due gonne

Son corrotte.

Vèndenn' giorn' e notte

Per una scoffia rossa,

Né mai lor ment' è scossa

Di far male.

A conferma, poi, dell'attitudine dell'autore a trasferire alla materia sacra strumenti espressivi della tradizione profana (nonché, nella fattispecie, della lirica amorosa), si aggiunga, infine, che questo stesso agile, duttile metro, dalla inconfondibile, resistente impronta popolareggiante, può una volta assumere la veste della lauda quando è imprestato a Helchia, il padre di Sosanna, per ringraziare il «Fattor de l'universo», che si è servito del profeta Daniele per palesare l'innocenza di Sosanna (a. IV, s. V, vv. I72-I79):

Le tue gratie son sparte

Hoggi sopra Sosanna

E sopra 'nnuoi, ch'Osanna

a te cantiamo

E 'l tuo nome lodiamo

Ch'è santo e gloriöoso.

Tu sei sempre riposo

ai tribulati. 
Il marito di Sosanna, Gioacchino, a sua volta, dialoga costantemente, più a lungo con sé stesso, e piuttosto gravemente in verità, in terza rima, il metro piu conveniente e autorizzato, oltre tutto, per esprimere il proprio sconforto nella scena IV dell'atto III, sul modello lirico e con tutti $i$ tópoi, appunto, di una disperata, dallinizio alla fine:

Ahimé dolente, quant’è mia disgratia!

Ahimé, dove son giont' e a qual partito!

Ahimé, che di dolor il cuor si stratia!

In tutto son, pur mo', son pur schernito!

Son fuor homai del dritto mio camino,

La mia fam' e l'honor hor è finito!

$[\ldots]$

O ciel, se gli è pietà la sù di sopra, Hor fa', ti prego, che da' monti cada

Qualche gran sasso che m'affoch' e copra.

Che 'l morir, più che vit' assai m'aggrada.

$E$ in terzine, quasi a voler marcare una distinzione anche metrica nei confronti del compagno Siro, nel senso di una minore giocondità e leggerezza, si esprime l'altro famulante, il meno sciolto e spiritoso Cleante, e in una parte di tanto minor rilievo. Quando poi accade che entrambi siano chiamati a dialogare fra loro, come nella scena II del III atto e nella $V$ del VI, $i$ due conservi, in verità, sembrano attuare una curiosa sorta di compromesso metrico, servendosi di stanze di 8 versi, alternatamente rotti e interi, settenari ed endecasillabi, secondo lo schema $\mathrm{aBaBaBcC}:{ }^{36}$ dove è degno di nota che nel dialogo concitato, per sticomitia, fra i pochissimi della tragedia, dei vv. 65-68 e 73-79 del primo esempio, e I82-203 del secondo, a Siro sia assegnata la misura 'rotta' e piacevole del settenario, a Cleante quella 'intera' e grave dell'endecasillabo.

Accade, naturalmente, che le esigenze del dialogo incidano talora, per eccezione, sulle prevalenti caratterizzazioni metriche degli interlocutori. Cosi nella scena IV del II atto a Siro potranno anche scappare di bocca poche terzine, dovendo concludere il dialogo e l'atto che Cleante aveva iniziato con quel metro; $e$ il terzinante Gioacchino nella scena $V$ dell'atto III si inserisce pur con qualche verso nelle ottave di Helchia ed 
Ethuria per poi scambiare con loro per intero anche qualche stanza (ma nella scena seconda del IV atto sarà Nachor a rinunciare al metro preferito e a dividere a sua volta col marito di Sosanna un paio di terzine). Ma il profilo metrico dei personaggi è in generale salvaguardato, in particolare per quanto riguarda la protagonista. Cosi in una scena capitale della tragedia, la II del II atto, Sosanna, disposta a «Patir tormento crudo assai più presto / E opprobrio manifesto, che mai faccia / Cosa, ch' a Dio dispiaccias, sgomitola la martellante sequenza delle sue rimalmezzo, contrapponendole con fermezza alle ottave subdole e insolenti con cui Nacchor e Chaynam tentano di vincere la sua resistenza ( $v v$. 67-76):

Levàtive de qua, malvaggi mostri,

Non son costumi nostri far tal cose,

Ma in opre virtuose e grat' a Dio

Mett'ogn'ingegno mio. Vecchi ribaldi,

Spegnete vostri caldi in meggio a l'acque

Dil mar, che sempre spiacque a Dio beato

Un sì enorme peccato, e più ne' Vecchi;

Che dovrst'esser specchi de virtute

E procurar salute alli altri tutti,

E vui più sète scelerat' e brutti. ${ }^{37}$

L'uscita a stampa della Sosanna precede di soli tre mesi quella romana della princeps della Sophonisba di Giangiorgio Trissino, composta una decina d'anni prima e già divulgata manoscritta. Restituendo all'etichetta di tragedia l'originario e classico significato drammaturgico, dedotto non soltanto dalla codifica zione teorica della Poetica aristotelica ma anche dal vaglio e dall' imitazione degli archetipi di Euripide e di Sofocle che Aldo Manuzio aveva portato pressoché integralmente alla luce delle stampe, lo scrittore vicentino faceva di colpo piazza pulita di tutti quanti i conatipiù o meno tragici che l'avevano preceduto, comprese le prove che abbiamo ricordato, proponendo un modello di tragedia che per quasi tre secoli avrebbe rappresentato un imprescindibile punto di riferimento per il teatro non solo italiano ma europeo. ${ }^{38}$ Un primato, il suo, tempestivamente riconosciuto, fra i primi da Giovan Battista Giraldi, nella coda in versi, La tragedia a chi legge, collocata alla fine della prima edizione dell'Orbecche (I543): 
E 'l Trissino gentil che col suo canto

Prima d'ognun dal Tebro e da l'Illisso

Già trasse la Tragedia a l'onde d'Arno. ${ }^{39}$

\section{Con la Sophonisba, ha scritto Carlo Dionisotti:}

l'Italia linguistica e letteraria del Trissino si affrontava all'antica Grecia direttamente, senza la mediazione romana. L'argomento stesso della tragedia, Sofonisba, esemplifica il distacco critico e l'avversione del Trissino nei confronti della tradizione romana..$^{\circ}$

Di là dalla scelta della fabula, l'oltranza grecheggiante, la norma classica, l'abito dell'erudizione costituiscono non soltanto il punto di partenza e la chiave di volta dell'esperimento trissiniano, ma anche, con le integrazioni e trasformazioni che via via si succederanno, la marca d'origine, il contrassegno distintivo, nonché l'accusato vizio d'origine per più secoli di storia della tragedia italiana.

Non meno che su quello linguistico, una parte cospicua della propria battaglia il caposcuola la gioca sul terreno metrico, sul duplice versante della riflessione teorica e dell'applicazione pratica. E stato ancora una volta Dionisotti a osservare come l'interesse teorico di Trissino per il teatro sia di gran lunga maggiore, non soltanto dell' abilità drammatica, ma anche «della fiducia che a torto o a ragione [egli aveva] nella propria abilità poetica e letteraria». ${ }^{4 \mathrm{I}}$ Più ancora delle divisioni della sua Arte Poetica, cronologicamente successive, importano, a questo riguardo, le considerazioni teoriche affidate alla lettera dedicatoria della Sophonisba presentata a Leone X nel Is I $8^{4^{2}}$ e poi premessa alla stampa del Is24, che anche costituisce, a giudizio di Bernard Weinberg, «the earliest extensive exploitation of the Poetics in Italian». ${ }^{43}$ Dal postulato aristotelico dell'unità della praxis drammatica dipende anche un passaggio decisivo della riforma, la reductio ad unum della varietà metrica che aveva talora caratterizzato anche le parti dialogiche dei precedenti tentativi tragici; e per quelle l'adozione dell'endecasillabo sciolto. ${ }^{44}$ In contrasto anche più accentuato e stridente con l'uso, riservato alle parte corali, delle forme liriche della tradizione, in primo luogo ma non soltanto petrarchesca, e non senza sintomatiche innovazioni, $^{45}$ la rivoluzionaria proposta era destinata a imporsi rapidamente, oltreché a inangurare il plurisecolare dibattito critico pro o contro la rima. ${ }^{46}$ 
Nella lettera dedicatoria della tragedia l'abbandono della rima è messo in relazione esplicita con un argomento capitale della Poetica aristotelica, investendo la stessa definizione di tragedia e la concezione della catarsi. Ancorché si tratti di una testimonianza notissima, non possiamo fare a meno di citarla, per la parte che qui interessa:

Quanto poi al non haver per tutto accordate le rime, non dirò altra ragione; perciò ch'io mi persuado che, se a Vostra Beatitudine non spiacerà di voler alquanto le orecchie a tal numero accommodare, che lo troverà e migliore $\mathrm{e}$ più nobile $\mathrm{e}$ forse men facile ad asseguire di quello che per aventura è reputato. E lo vederà non solamente ne le narrazioni et orazioni utilissimo, ma nel muover compassione necessario; perciò che quel sermone il quale suol muover questa, nasce dal dolore, et il dolore manda fuori non pensate parole, onde la rima, che pensamento dimostra, è veramente a la compassione contraria. ${ }^{47}$

Anche se nelle prime quattro divisioni della Poetica, pubblicate nel I529, il Trissino non parla esplicitamente di "verso sciolto", né del nome né della cosa ${ }^{48}$ questa giustificazione poggia, in verità, su un presupposto illustrato nel capitolo sulla rima che apre la seconda divisione, dove si insiste sull'autonomia, sull'autosufficienza ritmica del verso volgare, indipendentemente dall'accordo delle desinenze. Per il Trissino la rima non è di per sé un elemento normativo, una condizione necessaria e sufficiente del verso, della sua unità ritmica, non può, pertanto, ridursi alla sola accezione di omeoteleuto, vulgata quanto si voglia ma del tutto accessoria e secondaria. Dal De vulgari eloquentia e da Antonio di Tempo, esplicitamente chiamati in causa non senza parzialità e qualche forzatura, egli aveva dedotto, quanto gli bastava, che rima equivale a ritmo, e per provare il suo assunto ricorre a un curioso e tuttavia efficace paragone:

La rima è quello che i Greci dimandano rithmo et i Latini numero, laonde si può dire che rima, rithmo e numero siano quel medesimo. [...] Il rithmo, adunque, de la voce articulata è una risonanzia che risulta da certa quantità e qualità di sillabe, con ragione poste insieme e con ragione terminate; onde adviene che col variare de la quantità e qualità de le sillabe, e mutare la ragione del ponerle insieme e terminarle, si varia anchora il ritmo, il quale nasce sempre da quelle, sendo però diverso da loro; sì come di una quantità di legni, a certa guisa lavorati e con certa ragione posti insieme, si fa una galea; ma ad un'altra, e con altra ragione, 
si farà una nave; et un'altra uno grippo; le cui forme quantunque dipendano da la quantità, qualità et ordinazione di detti legnami, sono però cosa diversa da essi. ${ }^{49}$

Non sarà Girolamo Muzio a definire con un calembour $i$ versi sciolti «rime senza rime»? ${ }^{50}$

Liberandolo dunque dall'accordo obbligato delle desinenze, prima ancora di definirne le puntuali coordinate teoriche e illustrarne diffusamente la varia fenomenologia ritmica, l'autore della Sophonisba poteva sancire con ragionevole approssimazione e notevolissima lungimiranza la naturale corrispondenza del maggior verso italiano, l'endecasillabo piano - anzi il «trimetro scemo», per servirci di quella terminologia - col verso dialogico degli archetipi tragici greci, il trimetro giambico, assumendone naturalmente come modello il paradigma catalettico. All'irrinunciabile esigenza prosodica di una virtuale isocronia, prima ancora che alla scarsa padronanza dello strumento tecnico o al difetto della 'vena' poetica, dovremo dunque attribuire la biasimata povertà e fiacchezza ritmica dello sciolto trissiniano, la cui irreprensibile scansione giambica registra infatti la nettissima supremazia dell'endecasillabo con accenti sulla quarta e sulla sesta sillaba e con cesura dopo questa, in caso di parola tronca, o, per eccellenza, dopo la settima (molto più raramente dopo l'ottava): con una percentuale che sfiora, nella tragedia, il 75\% delle occorrenze; ${ }^{5}$ mentre si contano, fra le numerate, eccezionali trasgressioni, soltanto due endecasillabi del tipo cosiddetto 'dattilico', con percussioni sulla quarta e sulla settima sillaba. $^{52}$ Un verso discorsivo, di natura prosastica, dalle medesime caratteristiche che Aristotele aveva indicato a proposito del trimetro giambico: 53 argomento decisivo, quest'ultimo, per il successo dell'endecasillabo sciolto come verso drammatico. Il Trissino, preciserà Giovan Battista Giraldi,

compose la sua Sofonisba in quella maniera di versi ch'egli, prima di ogniuno, diede convenevolissimamente alla scena, in luogo del iambo ch'usano i Greci ed i Latini nelle scene. Però che parve a lui che la medesima ragione portassero con loro que' versi, sciolti dalla obligazion delle rime, che portavano anche i senarii composti de' iambi nella greca e nella latina lingua, cioè che fossero simigliantissimi al parlare famigliare de' nostri tempi e cadessero, come i iambi, dalla bocca de' favellatori (ancora ch'essi non vi pensassero) ne' communi ragionamenti. ${ }^{54}$ 
Un verso prosastico, capace di scendere alla «bassezza e trivialità del sermo pedestris, che Torquato Tasso avrebbe biasimato con fermo giudizio, contrapponendogli la «lirica dignità» dei cori, "più favoriti da l'ispirazione d'Apollo»." Is Il poeta tragico si trovava nella necessità di dover soddisfare due esigenze inevitabilmente contraddittorie e che pure gli apparivano conciliate in maniera insuperabile negli esemplari greci: l'altezza dello stile, da una parte, la dialogicità, la colloquialità, dall'altra. Una sfida ardua, forse impossibile, quando ci si trovava a dover adeguare il «superbissimum carmen», senza l'appoggio della rima $e$ in assenza di una tradizione specifica, alle rinnovate esigenze della scena e del coturno.

Renzo Cremante 
I. G. W. Most, Giangiorgio Trissino. La Sofonisba, in Palladio, a cura di G. Beltramini e H. Burns, Venezia, Marsilio, 2008, p. 33.

2. Anche se è ben ragionevole supporre che Trissino non potesse non essere, direttamente o indirettamente, a conoscenza dell'esperimento di Galeotto, le sparse consonanze fra le due Sofonishe, che pure è dato di riscontrare, saranno verosimilmente da riferire alla comune fonte liviana, spesso tradotta alla lettera dall'uno e dall'altro autore. Valga, fra i tanti, l'esempio delle parole pronunciate da Sofonisba, subito dopo aver ricevuto dal Servo di Massinissa la fatale coppa di veleno (c. 47v): «Il dono marital, ch'ei manda, accetto, / A me ben condecente, e non ingrato, / E tal lo stimo, qual con ogni affetto / Spettar si può dal buon marito amato, / Pur, come sarai gionto al suo conspetto, / Digli ch'il meglio per me fora stato / Esser inanzi morta, e con più requie, / Ch'essermi ad altri data in queste essequie» (e Trissino, Sof., I 586-9 I: «Al tuo Signor dirai / Che la sua nuova sposa volentieri / Accetta il primo don ch' a lei ne manda, / Poi che non le può dar cosa migliore. / Ver è che più le aggradiria il morire / Se ne la morte non prendea marito»), da confrontare con il discorso diretto riferito da Livio, XXX, i , 6-7: «Accipio -inquit- nuptiale munus, neque ingratum, si nihil maius vir uxori praestare potuit. Hoc tamen nuntia, melius me morituram fuisse si non in funere meo nupsissem». Per un riscontro più circostanziato si vedano, altrimenti, le note di commento all'edizione della Sophonisba di Trissino compresa in Teatro del Cinquecento. Tomo I. La tragedia, a cura di R. Cremante, Milano-Napoli, Ricciardi, 1988 (La letteratura italiana. Storia e testi), in particolare ai vv. 55-56, 77-79, 272-80, 390-408, 409-I7, I 28 I-86, I 57 I-83. A questa edizione saranno da riferire, d'ora in avanti, tutte le citazioni della tragedia (pur rinunciando, in questa sede, alla conservazione delle «lettere nuovamente aggiunte» della riforma ortografica trissiniana).

3. La Sophonisba tragedia del magnifico cavaliere e poeta messer Galeotto Carretto, In Vinegia, Appresso Gabriel Giolito de Ferrari, MDXLVI. Fin dal I 542, in una lettera allegata al Dialogo [...] Dove si ragiona delle Bellezze (Venetiis, Apud Antonium Gardane, MDXXXXII, c. I I 3v), lo stesso Niccolò Franco, tessendo le lodi di Galeotto, aveva invitato Alberto del Carretto, nipote del poeta e depositario delle sue carte, «a dar tosto a la luce non pur le Vertù Prigioniere, ma le Tre Comedie, la Sophonisba, le Rime de la vita Cortigiana e ciò che scrisse, percioché in ogni suo scritto parmi conoscere acutezza d'ingegno, novità di trovare, e destrezza di Satira: al cui soggetto, egli come nimico del vitio, parve attamente nato nel nostro secolo vitioso». Per la tragedia di Galeotto, pur in presenza di un'edizione moderna (Galeotto Del Carretto, Li sei contenti e La Sofonisba. Edizione e commento di M. Bregoli-Russo, Madrid, Ediciones José Porrúa Turranzas, 1982), sono costretto servirmi dell'unica stampa cinquecentesca. Sull'autore e sull'opera, tuttora fondamentale lo studio di G. Manacorda, Galeotto del Carretto poeta lirico e drammatico monferrino (I4.-I530), in «Memorie della R. Accademia delle Scienze di Torino», serie II, XLIX, I 898-1899, pp. 47-I 25. Oltre alla voce del DBI, firmata da Roberto Ricciardi, e alla sezione debitamente riservata a Galeotto in Teatro del Quattrocento: le corti padane, a cura di A. Tissoni Benvenuti e M. P. Mussini Sacchi, Torino, Unione tipografico-editrice torinese, I 983 , si vedano ancora: T. Cipriani, Sofonisba, tragedia di Galeotto del Carretto: studio, Catanzaro, Tip. G. Caliò, I9o6; G. Turba, Galeotto Del Carretto tra Casale e Mantova, in "Rinascimento», serie II, XI (I97I), pp. 95-I69; da ultimo, L. Rossi, "La Sofonisba" di Galeotto del Carretto, in «Levia Gravia», 2 (2000), pp. I 8 I-87. 
4. Proprio nel i 546, per esempio, vedono la luce l'Orazia di un oramai irriducibile avversario di Franco come l'antico suo protettore Pietro Aretino (anch'essa per gli stessi tipi di Giolito), due distinte edizioni della Canace di Sperone Speroni, e la tragedia anepigrafa e postuma del lucchese Giuseppe Baroncini (se non vogliamo considerare anche un testo eccentrico e dottrinale come il Libero arbitrio, in prosa, del riformato Francesco Negri, che pure si presenta, nel frontespizio, con la qualifica di «tragedia»).

\section{A quella data essa già contava non meno di 5 edizioni.}

6. Il Franco, mentre si guarda bene dal nominare esplicitamente lo scrittore vicentino, si preoccupa però di sottolineare a più riprese come le due tragedie siano entrambe destinate alla lettura: «亡̀ il vero dunque che la tragedia di Sophonisba è stata altre volte mostrata in rima, né perché, come dico, sia stata letta, resterà che hora dandosi a leggere in altre rime, se ne rimaranno i lettori»; ed ancora: «e questo per la novità almeno non potrà annoiare i lettori».

7. Nell'uso cinquecentesco, la definizione di “opera continuata" può ugualmente riferirsi sia a un poema, sia a un'opera storica. La lettera di dedica, inaugurando un tópos che sarà in seguito ripreso, tra gli altri, da Ludovico Dolce nel Prologo della Medea (I 557), non manca di mettere in relazione l'assunto tragico con la qualità dei tempi calamitosi, come per le «guerre d'Italia», così per la stessa vicenda personale dell'autore, costretto, proprio in quel tempo, all'esilio: «lodevole cosa è l'accomodarsi a' tempi, e secondo l'occorrenza de' casi hor lieti, hor tristi, saper dispensare il viver nostro con qualche esteriore segno [...]. Il perché veggendo questi tempi di guerre, di travagli e di mestitia e di mille altri infortuni pieni, per accomodarmi a quelli, mi parve di prendere uno assunto a questi giorni a tale occorrenza accomodato»; ricordando quindi con l'esempio di Sofonisba, ma pensando anche a sé stesso, «quanto è da stimare la bella e pietosa libertà, la quale né per oro, né per gemme, né manco per stati, si puol vendere né commutare» (c. 5r).

8. Nell'alta frequenza dei monologhi Manacorda ravvisa una possibile influenza dei Mystères francesi, nei quali appunto essi spesseggiano (Galeotto del Carretto, p. 93).

9. Corrispondenti ai libri XXVII-XXX delle Deche di Livio.

Io. Un carattere peculiare dell'azione scenica è rappresentato dalla molteplicità e mutevolezza delle scene, e dunque dall'incessante dinamismo, dal perpetuo moto dei personaggi, sicché molti dialoghi o monologhi sono pronunciati in itinere, come puntualmente dichiarano le didascalie: «Lelio solo per camino dice» (c. 6v); «Asdruballe conducendo Sophonisba le dice per camino» (c. 29r); «Lelio essendo partito da Scipione dice per camino» (c. 3or); «Lelio ritornando a Scipione, dice per strada» (c. 32v); «Asdruballe andando a Carthagine, dice per camino» (c. 33v); «Lelio venendo a Cyrtha dice per camino» (c. 42r); «Il servo per camino dice solo» (c. 47r); «Il nuntio di Massinissa uscendo da le porte, et andando al patrone, dice» (c. 49r). 
I I. La bipartizione lascia intravedere un'autorizzazione classica. La troviamo anche, per esempio, nella Pamphila del Pistoia, e più volte, naturalmente, nel percorso della tragedia cinquecentesca, dalla Dido in Cartagine di Alessandro Pazzi de' Medici, alla Tullia di Ludovico Martelli, all'Orbecche di Giovan Battista Giraldi, ecc.

I 2. Il mostro dell'Invidia è ugualmente esecrato dal Coro nella «canzonetta» che chiude il I atto della commedia Noze de Psiche et Cupidine dello stesso Galeotto (cfr. Teatro del Quattrocento, p. 637).

13. La Fede, per esempio (che qui impronta con una martellante ripetizione anaforica le prime otto terzine del Coro a commento della rottura dell'alleanza coi Romani e del «tradimento» di Siface), sarà parimenti invocata dal Coro nel IV atto dell'Orbecche: dal quale dipende, fra l'altro, il Coro, ugualmente del IV atto, della Giocasta di Ludovico Dolce.

I4. A c. $48 \mathrm{rv}$, per esempio, la terna di parole rima sorte : morte: corte è replicata a distanza di due sole ottave.

I 5 . Non mancano nemmeno, del resto, rari esempi di ottave capfinidas: cfr. c. 36v: «A spregiar di fortuna ogni disdetto. // Ogni disdetto dunque è da spregiare»; c. $45 \mathrm{v}$ : «Non saper se gli è honore, o s’è vergogna. // Vergogna esser conosco il darsi in mano»; c. 5ov: «Questo conforto sol mi fa minore / Il danno, e vivo tiemmi nel dolore. // Nel dolor tiemmi vivo il gran conforto».

I6. Il metro, là assumendo la denominazione di «oda», è collaudato dal Carretto anche nell'azione mitologica Noze de Psiche et Cupidine, composta nel I 499-I 500 e pubblicata nel I 520 , per la quale si rimanda alla citata edizione compresa nel Teatro del Quattrocento: atto I, vv. 26I-84, pp. 629-30, e vv. 347-70, p. 634; atto IV, vv. 423-46, pp. 704-705; atto V, vv. I 27-43, pp. 7I 2-I 3 (e cfr. anche F. Bausi e M. Martelli, La metrica italiana. Teoria e storia, Firenze, Le lettere, I993, p. I69); nonché nella commedia allegorica Tempio de amore, pubblicata nel i 58 (ma si veda ora l'edizione critica a cura di C. Caramaschi, introduzione di F. Magnani, Roma, La Fenice, I997). Di quest'ultima aveva già riportato e commentato un esempio R. Spongano, Nozioni ed esempi di metrica italiana, Bologna, Patron, I966, pp. 235-36. Ancora utile il capitolo dedicato alla varia tipologia dei metri lirici e drammatici nella citata monografia di Manacorda, Galeotto del Carretto, pp. 6i-7 I.

17. Sulla morfologia della frottola si veda, più in generale, S. Verhulst, La frottola (XIV-XV sec.): aspetti della codificazione e proposte esegetiche, Gent, Rijksuniversiteit Gent, I990.

I 8. Ma anche a formule ragionative del tipo: «Quinci creder si può» (c. 5 Ir, e cfr. Trissino, Sof., I 379: «Quinci si può veder»).

I 9. «L'altra sarà la passionale, come è lo Aiace, la Sofonisba, e simili, e questa arà il suo stato dal discorso, perciò che da esso si preparano le passioni, cioè la misericordia e la tema e l'altre similì (Trattati di poetica e retorica del Cinquecento, a cura di B. Weinberg, Bari, Laterza, I974, vol. II, p. 28). 


\section{Renzo Cremante}

20. Per una prima informazione di carattere generale, tuttora imprescindibili gli studi di Ferdinando Neri, dal I capitolo (Le tragedie in rima) e dai successivi del volume La tragedia italiana del Cinquecento, Firenze, Tipografia Galletti e Cocci, I 904, all'articolo, pur prevalentemente dedicato alla versificazione comica, Il verso drammatico (dal Nardi allo Stroz₹i), in "Giornale storico della letteratura italiana», CXIX (I 942), pp. I-3 I (quindi in Letteratura e leggende. Raccolta promossa dagli antichi allievi con un ritratto e la bibliografia degli scritti del Maestro, Torino, Chiantore, I95 I, pp. 78-1 Iо).

21. Tragedia nova intitolata Sosana raccolta da Daniello propheta, per Tibortio Sacco bussetano, Stampata in Vinegia, per Benedetto et Agostino, Fratelli de Bendoni. A dì XX de Aprile MDXXIIII. La tragedia dovette godere di una qualche fortuna cinquecentesca, se fu ancora ristampata nel 1537 a Brescia, per i tipi di Damiano di Turlini, mentre si ha ancora testimonianza di una sua rappresentazione nel I 553 . Ne ha procurato un'edizione criticamente accertata e sobriamente annotata - della quale ci serviamo, pur con qualche minimo ritocco - F. Magnani, La tragedia "Sosanna" di Tiburzio Sacco bussetano, in Parma e l'Umanesimo italiano. Atti del convegno internazionale di studi umanistici (Parma, 20 ottobre 1984), a cura di P. Medioli Masotti, Padova, Antenore, I986, pp. 173-230. Cfr. anche S. Cremonini, Sacre rappresentazioni al nord: riflessioni e presenze, in Boiardo e il teatro: $i$ cavalieri in scena. Atti del Congresso, Scandiano, i 5-I6 maggio 2009, a cura di G. Anceschi e W. Spaggiari, Novara, Interlinea, 2010, pp. IоI-27.

22. Magnani, La tragedia "Sosanna", pp. I 86-87.

23. Prologo, 26-28.

24. Prologo, 3I-36.

25. Si noti l'uso di una terminologia che appartiene anche al vocabolario tecnico della Poetica aristotelica.

26. Cfr. Magnani, La tragedia "Sosanna", p. 175.

27. Prologo, 4I-52.

28. Compresi il sonetto caudato in dialogo fra l'autore e la protagonista che fa da introduzione, il Prologo in ottave e l'Argomento, anch'esso in forma di sonetto caudato. Non eccezionale, nei testi teatrali quattro-cinquecenteschi, l'uso del sonetto, in particolare del sonetto caudato, per l'Argomento. Così, per esempio, nella Commedia dell'Ingratitudine di Giovanni Battista Dell'Ottonaio (I $482-$ I 527 ).

29. La contiguità cronologica della Rosmunda con la Sophonisba di Trissino è attestata da un noto aneddoto raccontato da Scipione Ammirato, Opuscoli. Tomo II. Con le tavole delle materie e cose più notabili, in Fiorenza, nella nuova Stamperia d'Amadore Massi e Lorenzo Landi, I637, p. 258.

30. La divisione in atti non è però osservata nel manoscritto apografo G. XI. 64 della Biblioteca Comunale di Siena - un codice di singolare importanza per la 
storia del teatro rinascimentale - dal quale dipendono le edizioni moderne della Rosmunda.

3I. Rispettivamente, 6 nel I atto, 4 nel II, 5 nel III e nel IV, 7 nel V.

32. Al servizio di Gioacchino, Siro ha un compagno, Cleante. Dalla tradizione comica i servi della Sosanna mutuano anche luoghi topici, come le ripetute lagnanze sulla propria condizione. Così per esempio Siro, a. I, s. III, vv. I72-79: «Non è già creatura, / In questo mondo nata, / Che sia più sgratiata / De nui servi. // Al fin giotti e protevi / Chiamati siamo tutti. / Son questi i nostri frutti / E le mercedil»; e Cleante, a. V, s. V, vv. I 53-58: «Non averò mai ben in questo mondo? / Ché, quando credo ripossarmi alquanto, / Allhor d'ogni fatica pur abbondo. // Io posso ben di questo darmi vanto: / Mai di poter posare una mezz'hora, / Ch'addoperato sono d'ogni canto», ecc. Di norma esclusa o diversamente connotata la figura del servo nella tragedia cinquecentesca d'imitazione classica, anche se, per esempio, ai colori diffusamente cortigiani della fabula, soprattutto, ficta sarà da addebitare la pur marginale presenza, nelle tragedie di Giraldi, di personaggi umili, 'comici' (e della relativa onomastica): servi, cameriere, segretari, soldati, sergenti, alfieri, eunuchi, paggi, perfino un Olimpo medico (Cleopatra) e una Pomilia nana (Euphimia).

33. Cfr. la c. zor. Di endecasillabi incatenati con rimalmezzo è anche il monologo - 34 vv. - di Catillo nella scena II del V atto della Commedia in versi di Lorenzo di Filippo Strozzi: cfr. l'edizione delle Commedie, a cura di Andrea Gareffi, Ravenna, Longo, I980, pp. 95-96. La commedia è ascrivibile al secondo decennio del sec. XVI.

34. Nelle Noze se ne servono, di volta in volta, Psiche, le sorelle e i loro mariti e infine, nell'ultimo atto, Giove, Venere, Mercurio e Cosmo. Con la differenza, tuttavia, che le serie di endecasillabi frottolati di Galeotto sono sempre introdotte da un verso settenario.

35. In modo che il quinario o il quadrisillabo formino, col settenario precedente, un endecasillabo con rimalmezzo. Così ricomposto il metro, sperimentato anche nella lirica cortigiana, è impiegato da Galeotto del Carretto, per esempio, nelle commedie Tempio de amore e Noze di Psiche et Cupidine. Si vedano in particolare, della seconda, le «canzonete» intonate dalle due sorelle di Psiche (vv. 74-85) e il Coro alla fine del secondo atto (vv. $4 \mathrm{I} 6-42)$, con schema $\mathrm{a}^{7} \mathrm{~b}^{7}\left(\mathrm{~b}^{7}\right) \mathrm{C}$ : si cita sempre dal Teatro del Quattrocento, p. 642, rispettivamente, e pp. 657-58; ed ancora da Eufrosino Bonini, nella commedia Iustitia, della quale si ipotizza una rappresentazione fiorentina del I 5 I 3 , cfr. Tre commedie fiorentine del primo 500 . Edizione critica e introduzione di L. Stefani. Prefazione di E. Faccioli, Ferrara-Roma, Gabriele Corbo Editore, I986, pp. I 34-35. Nei repertori settecenteschi (Crescimbeni, Quadrio, Affò), il metro è registrato sotto la voce «Zingaresca». Cfr. F. Magnani, La zingaresca: storia e testi di una forma, Parma, Zara, 1988.

36. Uno schema analogo, però con un endecasillabo al posto del settenario in settima posizione, ricorre anche, come si è sopra indicato, nella Sophonisba di Galeotto, c. I Iv. Un'ottava con schema AbAbAbCC è impiegata una volta da Ethuria in questa stessa Sosanna, a. IV, s. V, vv. i88-95. 
37. Le rimalmezzo della protagonista si confronteranno ancora con le ottave dei due Vecchi nella scena III del IV atto.

38. Valga per tutte la testimonianza settecentesca del Prologo premesso da Alexander Pope a The Tragedy of Sophonisba. Acted at the Theatre-Royal in DruryLane by His Majesty Servants, by Mr. [James] Thomson, London, Printed for A. Millar, at Buchanan's Head, over-against St. Clement's Church, in the Strand, MDCCXXX: «When learning, after the long Gothic night, / Fair, o'er the western world renew'd his light, / With arts arising, Sophonisba rose: / The tragic muse, returning, wept her woes; / With her th'Italian scene first learnt to flow. / Her charms the Gallic muses next inspir'd: / Corneille himself saw, wonder'd and was fir'd».

39. Si cita dall'edizione dell'Orbecche compresa nel volume Teatro del Cinquecento, p. 445. Il giudizio è riecheggiato da Ludovico Dolce nel Prologo dell'Ifigenia (Venezia, Giolito, I 5 I I c. 5 2r: è laTragedia che parla): «E come su l’'Ilisso / Stetti molti anni, così a me non piacque / D'abitar sopra il Tebro. Or sopra l'Arno / Volger mi fece il piede assai pomposa / Quel che già pianse il fin di Sofonisba».

40. L'Italia del Trissino, in Atti del Convegno di studi su Giangiorgio Trissino, Vicenza, 3 I marzo-i aprile i979, Odeo del Teatro Olimpico, a cura di N. Pozza, Vicenza, Accademia Olimpica, I980, p. 17 (quindi in Scritti di storia della letteratura italiana. III. I972-1998, a cura di T. Basile, V. Fera e S. Villari, Roma Edizioni di Storia e Letteratura, 2010, p. I 55 ).

4I. Nella recensione al volume di R. P. Horne, The Tragedies of Giambattista Cinthio Giraldi, London, Oxford University Press, 1962, in «Giornale storico della letteratura italiana», CLX (1963), p. i I6.

42. Cfr. B. Morsolin, Giangiorgio Trissino: Monografia d'un gentiluomo letterato nel secolo $X V I$, Firenze, Le Monnier, I $894^{2}$, p. I 55 .

43. B. Weinberg, A History of Literary Criticism in the Italian Renaissance, Chicago, The University of Chicago Press, i956, vol. I, p. 369.

44. Per la denominazione, il DELI rinvia a Varchi (ante I 575 , e cfr. A. Menichetti, Metrica italiana: Fondamenti metrici, prosodia, rima, Padova, Antenore, I993, p. I 20). Ma l'attestazione più antica a me nota appartiene a Lorenzo di Filippo STRozzi, nel Prologo della commedia La Pisana, vv. 24-25: «e, visto ch'era una commedia fatta / in prosa prima, e poi in versi sciolti, io [...]» (cfr. la citata edizione delle Commedie dello Strozzi, a cura di A. Gareffi, p. I 37). Rimasta inedita fino a questa edizione, la data di composizione della commedia è forse da assegnare al i 5 I 8. Fra le prime attestazioni a stampa di "verso sciolto", la protasi delle $A p i$, il poemetto di Giovanni Rucellai pubblicato postumo nel I 539 , quasi quindici anni dopo la morte dell'autore, vv. 24-25: «Non temerò cantare i vostri onori / Con verso etrusco da le rime sciolto».

45. Una tavola completa dei metri utilizzati nelle parti corali della Sophonisba è proposta da Vercingetorige Martignone, nel saggio menzionato nella nota 
seguente, da integrare con le note di commento dell'edizione della tragedia compresa in Teatro del Cinquecento.

46. Sulla sperimentazione metrica della Sophonisha trissiniana e sul dibattito cinquecentesco intorno al verso sciolto si vedano: J. M. Steadman, Verse without rime: Sixteenth-century Italian defences of "versi sciolti", in «Italica», XLI (1964), pp. 384-402; A. Daniele, I confini metrici del testo: Sull'endecasillabo sciolto nel Cinquecento, in Teoria e analisi del testo. Atti del $5^{\circ}$ convegno interuniversitario di studi (Bressanone 1977), a cura di D. Goldin, Padova, Cleup, I98 I, pp. I 2 I-34; M. Martelli, Le forme poetiche italiane dal Cinquecento ai nostri giorni, in Letteratura italiana, vol. III. Le forme del testo. I. Teoria e poesia, Torino, Einaudi, I984, pp. 5 I 9 sgg.; F. Bausi, "Imitar col canto chi parla": Verso sciolto e "recitar cantando" nell'estetica cinquecentesca, in "Bibliothèque d' Humanisme et Renaissance», LI (1989), n. 3, pp. 553-68; V. Martignone, Modelli metrici della tragedia cinquecentesca in rapporto con il "Torrismondo" tassiano, in «Studi tassiani», XXXVII (1989), n. 37, pp. 7-36; F. Bausi e M. Martelli, La metrica italiana: Teoria e storia, Firenze, Le Lettere, I993, pp. I 47 sgg.; R. Harneit, Dramatische Texte "en vers non rimez, mais libres"? Poetologische Reflexion über das Versproblem im italienischen Theater des Cinquecento und Honoré d'Urfés Vorwort zu La Sylvanire ou la Morte-Vive, in «Romanistisches Jahrbuch», 45 (1994), pp. 8 5-i i6; E. Selmi, Il dibattito retorico sul verso tragico nel Cinquecento, in Il verso tragico dal Cinquecento al Settecento. Atti del Convegno di Studi, Verona, I4-15 maggio 2003, a cura di G. Lonardi e S. Verdino, Padova, Esedra, 2005, pp. 63-104.

47. Teatro del Cinquecento, pp. 3 I-32. Non occorre sottolineare l'importanza, di ordine anche generale e teorico, dell'accostamento di «rima» e «pensamento». Fra i primi a impugnare l'argomento, spostando pro domo sua il discorso sulla qualità dei versi e sulla libertà metrica, Sperone Speroni, in una primitiva redazione delle Lezioni sopra $i$ Versi in difesa della Canace, rimasta inedita e fatta conoscere soltanto alcuni decenni fa. Cfr. Sperone Speroni, Canace e Scritti in sua difesa Giambattista Giraldi Cinzio, Scritti contro la Canace, Giudizio ed Epistola latina, a cura di C. Roaf, Bologna, Commissione per i testi di lingua, I982, p. 304: «Non si scorge il pensamento nelle rime, se non ove in un medesimo modo si rispondono, ma la libertà di esse lieva del verso tal suspizione. E ciò sia detto anche della varietà di esse rime e di essi versi, ché non sendo tutti di un numero di sillabe determinato, ma lunghi, brevi, mediocri, lievano il pensamento e par che a caso sian fatti tali».

48. La denominazione compare, una sola volta, nella sesta divisione, pubblicata postuma nel i 562 .

49. Si cita da Trattati di poetica e retorica del Cinquecento, a cura di B. Weinberg, vol. I, Bari, Laterza, i970, p. 44.

50. Dell'Arte Poetica, г 07 2- го8 г.

5 I. Delle cesure, «che hanno grandissima forza nei versi, e sono di grandissimo ornamento a chi le sa commodamente usare», tratta l'ultimo capitolo della seconda divisione della Poetica trissiniana (Trattati di poetica e retorica, vol. I, pp. 62-64). A giudizio del metricologo le cesure «compiute», e cioè dopo una parola piana che termini con la quinta o la settima sillaba, fanno «più bello e più 
sonoro il verso». Secondo il Neri, «a voler esser chiari, la metrica del Trissino è tutta qui nelle cesure, che rispondono poi alla considerazione comune pei versi volgari, dell'accento» (La tragedia italiana del Cinquecento, p. 30 ).

52. Cfr. i vv. I077 e I 582 (senza considerare il v. I 454, che tuttavia non è "sciolto" ma appartiene all'epodo di una canzone pindarica intonata dal Coro nel terzo stasimo). Rari, del pari, gli esempi di ripercussione dell'accento sulla VI e sulla VII sillaba. Si può aggiungere che poco più rilevante sembrerebbe, a una prima ricognizione, l'occorrenza dell'endecasillabo dattilico nell'Italia liberata.

53. Poetica, 4, 7: «fu la natura stessa a trovare il metro adatto, perché il trimetro è fra tutti il più discorsivo; e la prova è che nel nostro discorrere giornaliero ci capita di pronunziare trimetri molto spesso, ma esametri molto poco, e sentiamo di uscire dal ritmo prosastico» (ARistotele, Dell'arte poetica, a cura di C. Gallavotti, Milano, Fondazione Lorenzo Valla - Arnoldo Mondadori editore, I978 3 , p. I 5 ).

54. Lettera indirizzata nel I 54I a Ercole II d'Este, per confutare alcune critiche mosse alla sua tragedia Didone, in Giovan Battista Giraldi Cinzio, Carteggio, a cura di S. Villari, Messina, Sicania, I996, p. I 56. Cfr. anche Discorsi intorno al comporre rivisti dall'autore nell'esemplare ferrarese Cl. I go, a cura di S. Villari, Messina, Centro interdipartimentale di studi umanistici, 2002 (Biblioteca umanistica), pp. го0-103 е 253-6г. Sono giudizi largamente rripresi nel corso del Cinquecento. Cfr. per esempio MatTeo di SAn Martino, Le osservationi grammaticali e poetiche della lingua italiana. A cura di A. Sorella, con la collaborazione di A. Leone, S. Martella e L. Matarese, Pescara, Libreria dell'Università editrice, I999, pp. 252-53: «Dico adunque che essendo la comedia e tragedia scambievoli ragionamenti de i casi predetti intervenuti, la prosa propriamente gli conveniva. Pur gli antichi scrittori, per accrescerle alquanto di dignità, le ridussero in poemi, attribuendoli il verso iambico. Il quale, secondo Aristotele nella sua poetica, più è atto de gli altri a i ragionamenti quotidiani, per l'argomento che 'l medesimo ne fa, cioè che nel parlar impensatamente molti iambici si formano e rarissimi essametri. Ma per ricercar in questa lingua versi che come i iambici alla prosa si appressino, parermi dirò che vi si convengano i versi sciolti, i quali per le qualità loro donde esser dovrebbeno in ogni altro poema refutati, sì come quelli che senza ritegno de i piedi e senza concatenation di rime poco da la prosa differiscono, per le medesime disproportion loro esser debbeno ne i predetti poemi admessi». Anche a giudizio di Francesco Patrizi, liberare l'endecasillabo dall'obbligo della rima non significò «farlo dalla natura e dal parlar commune lontano, ma ben fu farvelo più vicino» (Sostentamenti del nuovo verso beroico, in L'Eridano in nuovo verso heroico [...]. Con $i$ sostentamenti del detto verso, In Ferrara, Appresso Francesco de Rossi da Valenza, I 557 , c. 3r).

55. La "Sofonisba" di Giangiorgio Trissino con note di Torq. ${ }^{\text {to }}$ Tasso edite a cura di Franco Paglierani, Bologna, presso Gaetano Romagnoli, i 884 (Scelta di curiosità letterarie inedite o rare dal secolo XIII al XVII. In appendice alla Collezione di Opere inedite o rare, Dispensa CCV). 